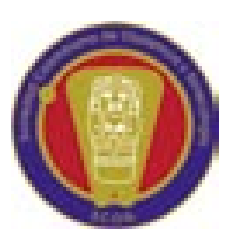

Revista Colombiana de Obstetricia y Ginecología Vol. 55 No.2 • 2004 • (129-135)

INVESTIGACIÓN ORIGINAL

ESTUDIO DESCRIPTIVO

\title{
CARACTERÍSTICAS GINECOLÓGICAS Y PSICOSOCIALES DEL DOLOR PÉLVICO CRÓNICO
}

\author{
PSYCHOLOGICAL, SOCIAL AND GYNECOLOGIC \\ CHARACTERISTICS OF CHIRONIC PELVIC PAIN
}

Claudia Patricia Criollo, M.D. ", Nora Myriam Romero, M.D. ., Olga Liliana Salazar, M.D. ", Freddy Briceño, M.D. ${ }^{* *}$, Hernando Navarro, M.D. ${ }^{* * *}$, Julián Alberto Herrera, M.D. ${ }^{* * * *}$

Recibido: marzo 4/2003 - Revisado: mayo 11/2003 - Aceptado: junio 25/2004

\section{RESUIMEN}

El dolor pélvico crónico es un síndrome prevalente controvertido en cuanto a su definición y a su relación con patología pélvica, problemas psicosocioculturales y su abordaje primario, diagnóstico y terapéutico.

Objetivo: describir el perfil sociodemográfico, las patologías orgánicas más frecuentemente observadas, y la asociación con síntomas de ansiedad y depresión en mujeres con dolor pélvico crónico en Cali, Colombia.

Métodos: se realizó un estudio descriptivo en mujeres con diagnóstico de dolor pélvico crónico que iban a ser sometidas a laparoscopia diagnóstica.

Resultados: se estudiaron 30 mujeres que cumplían los criterios de inclusión y aceptaron participar en el estudio. La edad promedio fue de 34,2 \pm 6,2 años con duración del dolor pélvico crónico entre uno y diez años. El 76\% de las mujeres vivían en pareja (casadas 53\%, unión libre 23\%), el 40\% pertenecían al estrato socioeconómico 3 (rango I-6), el 53\% eran de raza mestiza, el 50\% tenía escolaridad universitaria. Todas las pacientes presentaron patología pélvica en la laparoscopia; las más frecuentes

Residentes Tercer Año Medicina Familiar, Facultad de Salud, Universidad del Valle, Cali, Valle del Cauca, Colombia.

** Profesor Asistente, Departamento de Ginecología y Obstetricia, Facultad de Salud, Universidad del Valle.

*** Ginecólogo laparoscopista. Profesor Asistente, Departamento de Ginecología y Obstetricia, Facultad de Salud, Universidad del Valle.

***** Profesor Titular. Departamento de Medicina Familiar, Facultad de Salud, Universidad del Valle. fueron las adherencias 26,6\%, adenomiosis 20\%, endometriosis $10 \%$ y ovario poliquístico $10 \%$, con un 43,4\% de combinaciones de múltiples patologías. Se observaron adherencias densas en intestino y pared lateral en el 30\% de las pacientes. La ansiedad evaluada por la Escala de Ansiedad y Depresión en el Hospital presentó un porcentaje alto 30\%, en contraste con una baja proporción de depresión 3,3\%.

Conclusiones: todas las pacientes estudiadas con dolor pélvico crónico presentaron patología pélvica, principalmente adherencias densas en intestino y pared lateral 30\%, con una mayor proporción de ansiedad que depresión. Estas características ginecológicas y psicosociales son propias de la población estudiada y difieren de estudios reportados previamente en otros grupos poblacionales.

Palabras clave: dolor pélvico crónico, depresión, ansiedad, laparoscopia.

\section{SUMMARY}

Chronic pelvic pain (CPP) is one of the chronic pain syndromes where controversy exists in its definition, accompanying pelvic pathology, psychosocial and cultural associated factors, diagnosis, and therapeutic approaches.

Objective: To describe the socio-demographic profile of women with CPP, pelvic pathology by laparoscopy, and the association with anxiety or depressive symptoms in the woman.

Methods: A descriptive study was performed in thirty women with CPP and diagnostic laparoscopy. 
Results: The mean age was of $34,2+6,2$ years old. Long duration of the CPP (range 1-10 years) was observed. The most of the women $40 \%$ were of middle socioeconomic status (level 3, range 1-6), mixed ethnic backgrounds 53\%, university education 50\%, and stable marital state (married 53\%, common marriage in law 23\%. All women had pelvic pathology by laparoscopy. The most frequent were adherences $36,6 \%$, adenomyosis $20 \%$, endometriosis $10 \%$, polycystic ovary (10\%), and the remaining $43.4 \%$ were mixed pelvic pathologies. A high proportion of dense adherences with the intestine 30\% was observed. Anxiety, characterized by Hospital Anxiety Depression Scale (HADS), was observed in high proportion (30\%) as well, in contrast with a low proportion of depression 3,3\%.

Conclusion: The studied women had a high frequency of anxiety and low frequency of depression. All cases were associated with pelvic pathology with a high proportion (30\%) of dense adherences with the intestine. This gynecologic and psychosocial characteristics were different in relation to others populations.

Key words: chronic pelvic pain, depression, anxiety.

\section{INTRODUCCIÓN}

El dolor pélvico crónico (DPC) es uno de los síndromes de dolor crónico, en el cual existe controversia en cuanto a su definición, ${ }^{1,2}$ etiología, ${ }^{2-6}$ causas ginecológicas, ${ }^{7-10}$ no ginecológicas,,${ }^{11-}$ ${ }^{13}$ factores psicosocioculturales, ${ }^{14-20}$ además de su enfoque diagnóstico y terapéutico. ${ }^{5,7,16,20-26}$ A pesar de su alta prevalencia $12-14,7 \%,{ }^{1,2,6,22,23}$ no existe tampoco una clara aproximación de su abordaje primario en forma integral. Uno de los modelos de atención para este síndrome es el biopsicosocial, en el cual se propone que el dolor crónico y la incapacidad son el resultado de interrelaciones complejas entre estimulación sensorial, factores psicológicos y socioambientales en el que cada uno de estos componentes son capaces de influenciar a los otros. ${ }^{1,3,22}$
Con base en este modelo biopsicosocial, el presente trabajo pretende describir el perfil sociodemográfico de las mujeres con DPC que consultaron en una clínica de la ciudad de Cali, Colombia, las patologías orgánicas encontradas y la presencia de síntomas de ansiedad y depresión.

\section{MATERIALES Y MÉTODOS}

Se trata de un estudio descriptivo que incluyó pacientes que asistieron a una clínica privada de la ciudad de Cali, Centro Especializado en Infertilidad, referidas con diagnóstico clínico de DPC, ${ }^{1,2}$ quienes estaban programadas para laparoscopia diagnóstica y contestaron un instrumento precodificado que incluía la Escala de Depresión y ansiedad en el Hospital, ${ }^{28,29}$ previo consentimiento informado. Se incluyeron mujeres entre los 18 y 45 años, orientadas en tiempo, lugar y persona, alfabetas, con capacidad de autodiligenciar la encuesta. Se excluyeron mujeres con alteración del sensorio, las que no aceptaron participar voluntariamente en el estudio, las que estaban en proceso de duelo menor de seis meses o estaban tomando fármacos que producían síntomas similares a los de depresión. ${ }^{27}$

El tamaño de la muestra se calculó utilizando la fórmula que se usa para estudios descriptivos $(\mathrm{N}=$ (z/d) ${ }^{2}$ x p x q), para lo cual se tuvo en cuenta la prevalencia de pacientes de la población general que tienen DPC 10\%, que al mismo tiempo no presentan patología pélvica en su laparoscopia diagnóstica de acuerdo a estudios realizados previamente 6\% y que al mismo tiempo tienen diagnóstico clínico de depresión 2\%; ${ }^{1,2,2,27}$ se utilizó un margen de error del 5\% y un grado de confianza del $95 \%(\mathrm{z}=1,96)$ $(\mathrm{N}=30)$.

La Escala de Ansiedad y Depresión en el Hospital (HADS: Hospital Anxiety and Depression Scale) fue desarrollada por A. S. Zigmond y R. P. Snaith en 1983 para permitir a los clínicos e investigadores (con confianza y validez) tener una herramienta práctica para identificar y calificar las dos formas más comunes de alteraciones psicológicas en pacientes con enfermedades somáticas. La escala de 
catorce preguntas es fácil de administrar, es bien aceptada y permite detectar tanto ansiedad como depresión. Más de 17 estudios han mostrado su alta sensibilidad y especificidad para la detección de la ansiedad y la depresión. Cuando los resultados de la escala se encuentran entre 0 y 7 puntos en la caracterización de ansiedad o de depresión, se clasifica como negativa, entre 8 y 10 puntos es dudoso el diagnóstico, entre 11-21 puntos es positivo y se confirma el caso como de depresión o de ansiedad dependiendo de las respuestas a cada una de las evaluaciones. La duración del dolor se evaluó por años cumplidos y su intensidad si era permanente o intermitente con períodos de remisión. Los síntomas asociados se evaluaron por anamnesis realizada por el médico investigador mediante revisión por sistemas.

Las variables sociodemográficas se categorizaron por edad en años cumplidos, nivel socioeconómico según la clasificación del Departamento Administrativo Nacional de Estadística (DANE) (rango 1-6; muy bajo: 1 , bajo: 2 , medio: 3-4, alto: 5 , muy alto: 6), estado civil de acuerdo con su estado marital legalmente reconocido, raza con base en su etnia, escolaridad de acuerdo al cumplimiento de los estudios en los diferentes ciclos de enseñanza formal.

El análisis estadístico se realizó con el paquete estadístico Epistat 3.0 (Richardson, TX,USA). Se hizo análisis univariado utilizando proporciones y medidas de tendencia central. Se reportaron promedios cuando los datos mostraban una distribución estadísticamente normal (sin datos extremos) y medianas cuando los datos no mostraban una distribución estadísticamente normal (con datos extremos) con sus respectivas medidas de dispersión y rangos. Se evaluó si existía correlación entre la ansiedad y la depresión y los posibles factores que pudieran afectar esta asociación. Se utilizaron pruebas de significancia estadística; un valor $p<0,05$ se consideró como significante.

\section{RESULTADOS}

Se evaluaron 30 pacientes con DPC mediante laparoscopia diagnóstica desde el mes de junio a octubre de 2001. Al realizar el análisis univariado se encontró que el promedio de edad de las pacientes fue 34,2 \pm 6,2 años. La mayoría pertenecía al nivel socioeconómico medio (nivel 3, rango 1-6) 40\%, eran de raza mestiza 53\%, con escolaridad universitaria 50\% y unidas el 76\% (casadas 53\%, unión libre 23\%) (tabla 1). Todas las pacientes presentaban patología pélvica; las más frecuentes fueron las adherencias 26,6\%. Por otra parte, se encontraron adherencias densas al intestino y pared lateral en el $30 \%$ de las pacientes, adenomiosis 20\%, varicocele 12,5\%; con menor frecuencia la endometriosis 10\% y el ovario poliquístico 10\%; el 43.4\% restante lo constituyeron las combinaciones de múltiples patologías (tabla 2).

La HADS mostró una mediana de 8,5 puntos (rango 2-18); se observó un 30\% de mujeres con DPC con un puntaje en rango diagnóstico de ansiedad. En contraste, la evaluación de depresión mediante la HADS reveló una mediana de 4,5 puntos (rango 1-13), con sólo un 3,3\% de mujeres con DPC con un puntaje en rango diagnóstico de depresión.

\begin{tabular}{lcccc}
\multicolumn{5}{c}{ Tabla 1. Características sociodemográficas en 30 mujeres } \\
con dolor pélvico crónico & & \\
\hline \multirow{2}{*}{ Estado civil } & Soltera & Casada & Unión libre & Separada \\
& $4(13 \%)$ & $16(53 \%)$ & $7(23 \%)$ & $2(7 \%)$ \\
\hline Escolaridad & Primaria & Secundaria & Universitaria & \\
& $1(3 \%)$ & $13(43 \%)$ & $15(50 \%)$ & \\
\hline Raza & Negra & Blanca & Mestiza & \\
& $3(10 \%)$ & $10(33 \%)$ & $16(53 \%)$ & \\
\hline Estrato socioeconómico & Nivel II & Nivel III & Nivel IV & Nivel V \\
& $7(23 \%)$ & $12(40 \%)$ & $6(20 \%)$ & $4(13 \%)$ \\
\hline
\end{tabular}


Tabla 2. Diagnósticos ginecológicos mediante

videolaparoscopia en 30 mujeres

con dolor pélvico crónico

\begin{tabular}{lcc} 
Diagnósticos & Número & Porcentaje \\
\hline Adherencias & 8 & 26,6 \\
\hline Adenomiosis & 6 & 20 \\
\hline Endometriosis & 3 & 10 \\
\hline Ovario poliquístico & 3 & 10 \\
\hline Endometriosis + adherencias & 3 & 10 \\
\hline Endometriosis + varicocele & 1 & 3,30 \\
\hline Endometriosis + Adenomiosis & 1 & 3,30 \\
\hline Endometriosis + otros & 1 & 3,30 \\
\hline Adherencias + varicocele & 1 & 3,30 \\
\hline Varicocele + EPI & 1 & 3,30 \\
\hline Varicocele + ovario poliquístico & 1 & 3,30 \\
\hline Varicocele + adenomiosis & 1 & 3,30 \\
\hline TOTAL & 30 & $100 \%$ \\
\hline
\end{tabular}

En cuanto a la duración del dolor, un 17\% de mujeres manifestaron que tenían menos de un año con dolor, 33\% de uno a dos años y medio, 17\% de dos y medio a cinco años, 22\% de cinco a diez años y un $11 \%$ más de diez años. Al analizar las características del dolor encontramos que el 75\% presentaban dolor cíclico y el 25\% dolor continuo.

En relación con los síntomas asociados encontramos que el 63\% no presentaban síntomas asociados, el 25\% mostraban síntomas ginecológicos como leucorrea e irregularidades menstruales, el 8\% síntomas gastrointestinales como dismotilidad y el $4 \%$ otros síntomas como cefalea.

$\mathrm{Al}$ revisar si las pacientes presentaban laparoscopias previas se encontró que el 27\% de ellas tenían al menos una laparoscopia previa indicada en la mayoría de los casos por endometriosis. El 20\% estaban histerectomizadas por miomatosis como primera causa. También se encontró que el 63\% tenían cirugías previas, entre las cuales se destacan cesárea y Pomeroy como causas predominantes.
Al hacer el análisis de correlación lineal y correlación múltiple se observó correlación positiva entre los puntajes de depresión y los puntajes de ansiedad ( $\mathrm{r}$ $=0,60, p=0,005)$ y entre los puntajes de ansiedad, depresión y edad $(\mathrm{r}=0,62$, $p=0,001)$. La edad fue una variable de confusión que no mostró un efecto independiente $(\mathrm{p}=0,87)$.

\section{DISCUSIÓN}

En los estudios realizados en otros países el promedio de edad observado de mujeres con DPC es de 27 a 29 años; ${ }^{1}$ en el presente estudio observamos un promedio de edad mayor. Las características sociodemográficas de las pacientes estudiadas fueron similares a lo reportado en estudios previos, especialmente en la etnia y el nivel socioeconómico, ${ }^{1}$ debido a que las mujeres incluidas en el estudio podían acceder a una evaluación laparoscópica por tener régimen contributivo y plan complementario de salud. La mayoría pertenecían al nivel socioeconómico medio; sin embargo, el 50\% de mujeres incluidas en el estudio tenían educación superior y el 76\% unión estable (matrimonio, unión libre), características que difieren de la población general colombiana. Es posible que la unión estable en este grupo de mujeres con DPC se haya comportado como una red de apoyo social para antagonizar los efectos negativos del estrés y disminuir el riesgo de depresión.

La endometriosis es una enfermedad progresiva que afecta del 5 al 10\% de las mujeres. Puede ser causa de DPC cíclico y no cíclico (dismenorrea y dispareunia profunda) ${ }^{30}$ y de infertilidad. ${ }^{1,7,8,10} \mathrm{El}$ diagnóstico definitivo se da por estudio histopatológico, con una adecuada correlación por visualización directa bien sea por laparotomía o idealmente por laparoscopia. Alrededor del 24\% de las mujeres que se quejan de DPC tienen esta patología. ${ }^{10}$ En este estudio se encontró que el 10\% de las pacientes con 
DPC presentaban endometriosis a la laparoscopia sin diferencia en lo reportado en la literatura.

Las adherencias constituyen uno de los hallazgos orgánicos más frecuentes detectados en el momento en que se practica una laparoscopia diagnóstica para evaluar el DPC en aproximadamente un 89\%. Tienen más prevalencia en mujeres con dolor crónico, aunque aparece con frecuencia y a veces en gran medida sin DPC. ${ }^{1,6}$ La adhesiolisis en pacientes con DPC no mejora el dolor, solamente en caso de adherencias densas que comprometen el intestino y la pared lateral. Las adherencias suelen ser consecuencia de alguna intervención quirúrgica previa, pero también pueden aparecer después de procesos inflamatorios como EPI, quistes rotos e infección posparto. ${ }^{6}$

La adenomiosis se sospecha por las características macroscópicas del útero a la laparoscopia, permitiendo hacer el diagnóstico presuntivo, ya que el definitivo se realiza por estudio histopatológico; es una enfermedad cuyos síntomas incluyen hipermenorrea, dismenorrea, dispareunia, dolor pélvico después de la menstruación y con el ejercicio vigoroso. Nuestros resultados están acordes con la literatura en cuanto a la correlación clínica entre DPC tipo cíclico y el hallazgo de endometriosis y adenomiosis.

El diagnóstico de síndrome de congestión pélvica se ha vinculado con várices y dolor pélvico en mujeres sin identificar una causa anormal evidente de dolor. Por venografía pélvica se ha demostrado que más del 80\% de las mujeres sin una causa manifiesta de dolor en la laparoscopia tienen congestión venosa intensa en las principales venas gonadales, que a menudo muestran una dilatación tres veces mayor que su diámetro normal. Por medio de la venografía transuterina, el 91\% de las mujeres con DPC han presentado signos de varicocele en comparación con el 10\% observado en las pacientes estudiadas. El dolor se describe como una molestia sorda e intensa en la pelvis que empeora al caminar, con una posición de pie prolongada y postcoito, que mejora con el reposo y el uso de AINES. ${ }^{6,30,31}$ El varicocele se asocia con insuficiencia venosa en la cara posterior del muslo y del periné, además de anomalías genéticas o perturbaciones en la estructura del colágeno de la pared venosa.

Por otra parte, el aumento en la irrigación arterial por procesos inflamatorios crónicos como en el caso de la EPI ${ }^{9}{ }^{11}$ puede generar un síndrome de congestión pélvica. De igual manera, los estados hiperestrogénicos como el síndrome de ovario poliquístico se asocia a una alteración del tono en la vasculatura venosa, favoreciendo la aparición de síntomas por congestión. Por lo tanto, el empleo de medicamentos que producen estados hipoestrogénicos como los análogos de GnRH, los progestágenos o antiestrógenos se asocian con disminución del calibre venoso y de los síntomas. ${ }^{32,33}$

Las reacciones al dolor son de índole afectiva, cognoscitiva y conductual. Los componentes afectivos son miedo, depresión, angustia, irritabilidad e ira. La depresión tiene una prevalencia en la población general del 3-5\%, ${ }^{27}$ otros estudios sugieren cifras del 10-25\% en mujeres y 5-12\% en hombres. En la consulta médica puede ser tan común como el 25\% de los pacientes. El 60\% de mujeres con DPC se asocian con diagnósticos psicológicos, especialmente los trastornos afectivos que incluyen la depresión en un 25 - 50\% y la ansiedad en un 10 - 20\% entre otros. ${ }^{1}$

En este estudio se encontró una prevalencia de ansiedad de un 30\% y de depresión de un 3,3\%, lo cual representa una proporción alta de ansiedad y una proporción baja de depresión, en comparación con lo reportado en la literatura. Esta baja prevalencia de depresión puede estar influenciada aparentemente por el apoyo social que proporciona la unión estable que tenían las mujeres incluidas en el estudio, al igual que su alta escolaridad (50\% universitaria), que contrasta con la proporción de mujeres con educación superior en la población general que ha sido reportada en un 15\% (Encuesta Nacional de Demografía y Salud, PROFAMILIA, 2000). ${ }^{34}$ Las pacientes de este estudio se clasifican como adultos jóvenes en su ciclo vital individual, 
donde la prevalencia de trastornos afectivos es menor comparada con otros grupos (ejemplo, adultos mayores 10\%). También se podría explicar la menor prevalencia de depresión probablemente por el menor tiempo de duración de dolor reportado por las pacientes del estudio, en comparación con lo reportado en la literatura. Se piensa que las pacientes con DPC ven en la laparoscopia una expectativa de solución de su problema, lo cual podría generarles mayor ansiedad.

Se encontraron niveles bajos de depresión y la causa del DPC en todas las mujeres tenía origen orgánico, resultados que difieren con lo reportado en otras poblaciones. La duración de los síntomas en este estudio tuvo un rango entre siete meses y más de diez años; en la mayoría de los casos se observó una duración entre 1 a 2,5 años similar al promedio reportado en otras poblaciones (2,5 años). ${ }^{1}$

Los desórdenes de dolor cíclico en mujeres con DPC contribuyen a la sintomatología en un 20$50 \% .{ }^{1,2}$ En este estudio se encontró el dolor cíclico en un $75 \%$ de las pacientes, en contraste con lo reportado en la literatura .

Los desórdenes de dismotilidad crónica representan el síntoma más común en pacientes con DPC sin patología ginecológica hasta en el 50\% de los casos; ${ }^{6}$ en esta investigación se encontró el síndrome de intestino irritable en un 8\% de las pacientes mucho más bajo que el reportado en la literatura.

El hallazgo de patología pélvica en la laparoscopia no tiene una relación implícita de causalidad por lo que se hace importante continuar con el seguimiento a largo plazo. De acuerdo con los resultados del estudio, las mujeres con DPC observaron altos niveles de ansiedad en una alta proporción asociada a patología orgánica, principalmente con adherencias densas al intestino y pared lateral, resultados que difieren con lo reportado en otras poblaciones.

Dentro del enfoque integral de la mujer con DPC es importante la evaluación e intervención de los aspectos emocionales, los cuales pueden estar asociados como consecuencia de su patología crónica. Se conoce cómo interactúan los factores psicosociales con las causas orgánicas y aumentan la sujetividad al dolor, lo cual magnifica el dolor en mujeres con DPC., 14, 20, 22 En la medida en que las pacientes tengan una evaluación integral por un equipo multidisciplinario es alta la probabilidad de hallazgos potencialmente causales de DPC en la laparoscopia.

\section{CONCLUISIÓN}

Todas las pacientes estudiadas con DPC presentaron patología pélvica, principalmente adherencias densas en intestino y pared lateral 30\% con una mayor proporción de ansiedad que depresión. Estas características ginecológicas y psicosociales son propias de la población estudiada y difieren de estudios reportados previamente en otros grupos poblacionales.

\section{REFERENCIAS}

1. Reitter R. Evidence-based management of chronic pelvic pain. Clin Obstet Gynecol 1998;41:422-35.

2. Zondervan K, Yudkin PL, Vessey M, Dawes MG, Barlow $\mathrm{DH}$, Kennedy SH. The prevalence of chronic pelvic pain in women in the United Kingdom: a systematic review. Br J Obstet Gynaecol 1998;105:93-9.

3. Rosenfeld JA. Chronic pelvic pain: an integrated approach. Am Fam Physician 1996;54:2187-8.

4. Toth P, Jothivijayarani A. Gynecology chronic pelvic pain. Chapter 7. University of Iowa Family Practice Handbook, $3^{\text {rd }}$ edition. Iowa; 2000.

5. American College of Obstetricians and Gynecologists. Chronic pelvic pain. ACOG Technical Bulletin No. 223. Washington, DC: ACOG; 1996.

6. Steege JF. Dolor pélvico crónico. Primera Edición. México, DF: McGraw-Hill Companies; 2000.

7. Farquhar C. Extracts from the "clinical evidence". Endometriosis. BMJ 2000;320:1449-52.

8. Ballweg M. Blaming the victim. The psychologizing of endometriosis. Obstet Gynecol Clin North Am 1997;24:441-53.

9. Miller D. Keep a low threshold in diagnosing PID. Patient Care 2000;:75-90.

10. Wellbery C. Diagnosis and treatment of endometriosis. Am Fam Physician 1999;60:1753-68.

11. Valencia P. EPI y otras causas de dolor pélvico crónico. Hosp Pract 2000;4:63-6. 
12. Clemons JL, Arya LA, Myers DL. Diagnosing interstitial cystitis in women with chronic pelvic pain. Obstet Gynecol 2002;100:337-41.

13. Sullivan M. DSM-IV Pain disorder: a case against the diagnosis. Int Rev Psychiatry 2000;12:91-8.

14. Elliott ML. Chronic pelvic pain: What are the psychological considerations? APS Bulletin 1996;6:12-5.

15. Reed BD, Punch MR, Roth RS, Gorenflo DW, Gillespie BW. Psychosocial and sexual functioning in women with vulvodynia and chronic pelvic pain, a comparative evaluation. J Reprod Med 2000;45:624-32.

16. Bodden-Heidrich R, Kuppers V, Beckmann MW, Ozornek MH, Rechenberger I, Bender HG. Psychosomatic aspects of vulvodynia. Comparison with the chronic pelvic pain syndrome. J Reprod Med 1999;44:411-6.

17. Edwards L, Mason M, Phillips M, Norton J, Boyle M. Childhood sexual and physical abuse. Incidence in patients with vulvodynia. J Reprod Med 1997;42:135-9.

18. Jamieson D, Steege J. The association of sexual abuse with pelvic pain complaints in a primary care population. Am J Obstet Gynecol 1997;177:1408-12.

19. Rose V. ACOG releases a statement an identification and treatment of adolescent victims of sexual assault. Am Fam Physician 1999;59:1688.

20. Savidge CJ, Slade P. Psychological aspects of chronic pelvic pain. J Psychosom Res 1997;42:433-4.

21. Stones RW, Mountfield J. Interventions for treating chronic pelvic pain in women. The Cochrane Library; 2002.

22. Doyle DF, Li TC, Richmond MN. The prevalence of continuing chronic pelvic pain following a negative laparoscopy. J Obstet Gynaecol 1998;18:252-5.
23. Arguello RA. Dolor pélvico crónico indicador de laparoscopia. Controversias en Ginecología y Obstetricia 2000;6:17-25.

24. Elcombe S, Gath D, Day H. The psychological effects of laparoscopy on women with chronic pelvic pain. Psychol Med 1997;27:1041-50.

25. Miller BA, et al. Five reasons to consider a hysterectomy. Patient Care 1999;33:34-50.

26. Carter JE. Surgical treatment for chronic pelvic pain. JSLS 1998;2:129-39.

27. Toro RJ, Yepes LE. Trastornos depresivos. Psiquiatría, Fundamentos de Medicina. $3^{\text {a }}$ edición. Medellín: CIB; 1997. p. 132-3.

28. Zigmond AS, Snaith RP. The Hospital Anxiety and Depression Scale. Acta Psychiatr Scand 1983;67:36170.

29.Herrmann C. International experiences with the Hospital Anxiety and Depression Scale-- a review of validation data and clinical results. J Psychosom Res 1997;42:17-41.

30. Heim LT. Evaluation and differential diagnosis of dyspareunia. Am Fam Physyician 2001;63:1535-43.

31. Gómez G. Varicocelectomía por laparoscopia: descripción de una nueva técnica y su acción sobre el dolor pélvico crónico. Med Reprod 1998;1:14-7.

32. Navarro H. Dolor pélvico crónico. En: Cifuentes R. (ed). Ginecología y obstetricia basadas en las evidencias. Bogotá: Editorial Distribuna; 2002. p.659-70.

33. Soysal M. A randomized controlled trial of goserelin and medroxyprogesterone acetate in the treatment of pelvic congestion. Hum Reprod 2001;16:931-9.

34. Profamilia. Salud sexual y reproductiva en Colombia. Encuesta nacional de demografía y salud. 2000.

35. Kaplan H, Sadock B. Comprehensive textbook of Psychiatry. VI. Baltimore: William \& Wilkins; 1995. 